\title{
Facial dimensions, bite force and masticatory muscle thickness in preschool children with functional posterior crossbite
}

\author{
Paula Midori Castelo(a) \\ Leonardo Rigoldi Bonjardim(b) \\ Luciano José Pereira ${ }^{(c)}$ \\ Maria Beatriz Duarte Gavião(d)
}

(a) Postdoctoral Student; (d) Professor Department of Pediatric Dentistry,

Piracicaba Dental School, State University of Campinas, Piracicaba, São Paulo, Brazil.

(b) Assistant Professor, Department of Physiology, Federal University of Sergipe, Aracajú, Sergipe, Brazil.

(c) Assistant Professor, Vale do Rio Verde University, Três Corações, Minas Gerais, Brazil.

\author{
Corresponding author: \\ Maria Beatriz Duarte Gavião \\ Faculdade de Odontologia de Piracicaba, \\ UNICAMP \\ Departamento de Odontologia Infantil, \\ Área de Odontopediatria \\ Av. Limeira, 901 \\ Piracicaba - SP - Brasil \\ CEP: 13414-903 \\ E-mail:mbgaviao@fop.unicamp.br
}

Received for publication on May 15, 2006

Accepted for publication on Jun 28, 2007

\begin{abstract}
Posterior crossbite may affect craniofacial growth and development. Thus, this study aimed to associate facial dimensions (by standardized frontal photographs) to masseter and anterior portion of the temporal muscle thickness (by ultrasonography) and maximal bilateral bite force in 49 children with deciduous and early mixed dentitions. They were distributed in four groups: deciduous-normal occlusion ( $\mathrm{DNO}, \mathrm{n}=15)$, deciduous-crossbite ( $\mathrm{DCB}, \mathrm{n}=10)$, mixed-normal occlusion $(\mathrm{MNO}, \mathrm{n}=13)$ and mixed-crossbite $(\mathrm{MCB}, \mathrm{n}=11)$. Anterior facial height (AFH), bizygomatic width (FWB), and intergonial width (FWI) were determined and associated with muscle thickness and bite force, applying Pearson's coefficients and multiple logistic regression, with age, gender, body weight and height as the covariates. FWB and FWI were correlated positively with the masseter thickness, whereas AFH/FWB and $\mathrm{AFH} / \mathrm{FWI}$ ratios had negative correlation, except in the DNO group. The correlation between $\mathrm{AFH} / \mathrm{FWB}$ and bite force in the MCB group was significantly negative. A higher AFH/FWB in MNO and MCB led to a significantly higher probability for functional crossbite development. In the studied sample, it was observed that children in the early mixed dentition with a long-face trend showed lower bite force and higher probability to present functional posterior crossbite, without significant influence of the covariates.
\end{abstract}

Descriptors: Masseter muscle; Temporal muscle; Bite Force; Face; Malocclusion. 


\section{Introduction}

Unilateral posterior crossbite is a malocclusion that usually develops in the deciduous dentition and may be related with occlusal interferences (functional pattern) or a skeletal problem. Past studies showed asymmetry in masticatory muscle activity and thickness, ${ }^{1}$ decrease in bite force magnitude, a smaller number of teeth in contact, ${ }^{2}$ and asymmetric jaw motion and growth of the orofacial structures ${ }^{3-6}$ in children with crossbite.

The interaction among bite force, jaw muscle size, and craniofacial morphology has been studied in adults and growing individuals, but those that involved young children are rare and the respective correlations are less evident. Craniofacial morphology has been evaluated by means of anthropometric measurements, ${ }^{7-9}$ cephalometrics, ${ }^{3,10,11}$ and facial photographs. ${ }^{8,12,13}$ Using frontal photographs, ultrasonography (US) and bite force measurements, subjects with a long-face pattern showed a thin masseter and a smaller bite force. ${ }^{12,13}$ Both, US and standardized photographs, have demonstrated to be reliable and secure methods without cumulative radiation effects. ${ }^{13,14}$

The decisions regarding timing, duration and prognosis of the treatment for malocclusions should be based on the knowledge of the growth and function of mandible, maxilla and other craniofacial structures, ${ }^{3,15}$ considering extrinsic etiological factors. ${ }^{716}$ Occlusal alterations that lead to mandibular displacement may result in a compensatory asymmetrical growth and long-term effects on the orofacial development. ${ }^{3,6}$ Thus, this study aimed to investigate the relationship among facial dimensions, masticatory muscle thickness and bite force in deciduous and early mixed dentitions in the presence of functional posterior crossbite. Age, gender, body weight and height were considered as covariates.

\section{Material and Methods}

The sample comprised 49 children, aged 42-84 months ( 26 boys and 23 girls). All children and their parents consented to participate in the study, which was approved by the Ethics Committee, Piracicaba Dental School, State University of Campinas, Piracicaba, SP, Brazil. They were selected after com- plete anamnesis and clinical examination, assessing body weight and height, morphological occlusion, dentition stage and presence of functional unilateral posterior crossbite or a crossbite due to dental inclination (both involving canine and deciduous molars). ${ }^{2}$ Children with bilateral skeletal crossbite, tooth anomalies of form, structure or number, oral tissue alterations, craniofacial abnormalities, severe obstruction of upper airways, and systemic muscle or joint disorders were excluded.

For recordings of muscle thickness, the dental arches were divided into right and left (normal occlusion groups) and normal and crossbite (crossbite groups) sides, being the crossbite independent of the dental arch side (left or right). The sample was distributed in four groups: DNO, deciduous-normal occlusion $(\mathrm{n}=15)$; DCB, deciduous-crossbite $(\mathrm{n}=10)$; MNO, mixed-normal occlusion $(\mathrm{n}=13)$; and MCB, mixed-crossbite $(n=11)$ (for gender distribution, see Table 2). All assessments and measurements were performed by the first author (PMC), and described in details by Castelo ${ }^{17}(2007)$.

\section{Masticatory muscle thickness measurement}

The masseter and anterior portion of the temporal muscle thickness were measured bilaterally by US (Just Vision Toshiba ${ }^{\mathrm{TM}}, 56 \mathrm{~mm} / 10 \mathrm{MHz}$ linear transducer, Otawara, Japan). Briefly, children were seated in an upright position with their heads in natural position; recordings were performed with the muscle relaxed (resting) and in maximal intercuspation. The measurements were determined three times, directly on the screen (accuracy of $0.1 \mathrm{~mm}$ ), and the values were averaged. The recording site was established by palpation, following the orientations: masseter - level halfway between the zygomatic arch and gonial angle; anterior portion of temporal - in front of the anterior border of the hairline. The transducer was placed perpendicular to the muscle fiber direction, using an air-tight inert gel on the skin surface, and moved gradually to obtain optimal visualization.

\section{Bite force measurement}

Maximal bilateral bite force was assessed with 
a flexible pressurized tube (diameter of $10 \mathrm{~mm}$ ) connected to a sensor (MPX5700 Motorola, Austin, TX, USA). Children were seated in an upright position with the head in natural posture and the tube was placed bilaterally on the deciduous molars; they were instructed to bite the tube as forcefully as possible. The minimum (initial pressure) and maximum values were obtained in pounds per square inch (PSI), and the difference between them was calculated for three evaluations and the final values were the respective means (accuracy of $0.1 \mathrm{~N})$. PSI was converted into Newton $(\mathrm{N})$, taking into account the tube area (force $=$ pressure $\mathrm{x}$ area).

\section{Facial dimensions}

Facial dimensions were determined by measuring standardized frontal photographs $(10 \times 15 \mathrm{~cm})$, taken with the child standing up at $1.05 \mathrm{~m}$ from the camera, teeth in maximal intercuspation and the Frankfort plan approximately parallel to the floor. The anterior facial height (AFH), bizygomatic facial width (FWB), and intergonial width (FWI) were handily traced on acetate paper and measured with a digital caliper (accuracy of $0.01 \mathrm{~mm}$ ). AFH/FWB and AFH/FWI ratios were calculated. The bisectrix of the angle between the mandibular and mandibular ramus planes determined the gonion point $(\mathrm{Go})^{8,12,13}$ (Figures 1A and B).
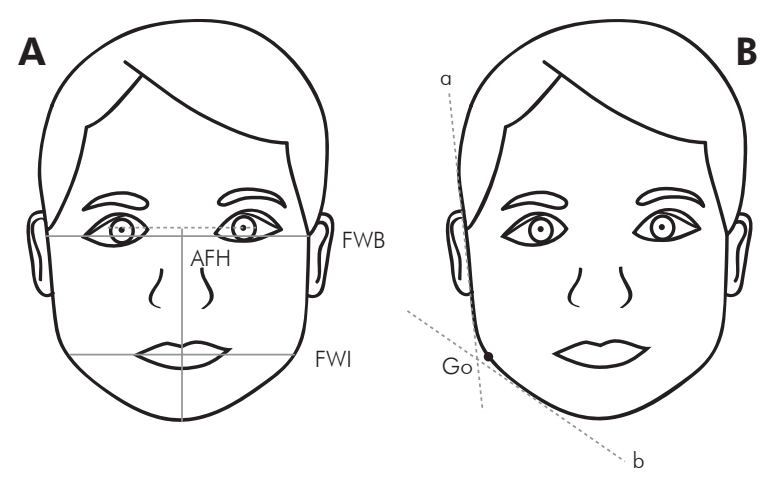

Figure 1 - Facial dimensions. AFH - anterior facial height, FWB - bizygomatic facial width, FWI - intergonial width (A). Gonion point (Go) determination. a - mandibular ramus plane, $b$ - mandibular plane (B).

\section{Statistical analysis}

Data from each dentition stage was separately analyzed. Pearson's correlation was used to determine the relative contribution of facial dimensions to muscle thickness and bite force. Multivariate logistic regression analysis with the binary endpoint of crossbite (yes, no) was fit to evaluate the association between presence of a functional posterior crossbite with facial $\mathrm{AFH} / \mathrm{FWB}$ and $\mathrm{AFH} / \mathrm{FWI}$ ratios, using a stepwise variable selection method to identify a set of measurements that were independently associated with presence of functional posterior crossbite, after adjusting for age, gender, body weight and height (Intercooled Stata 7.0, College Road, TX, USA).

\section{Results}

The descriptive statistics are shown on Tables 1 and 2. Muscle thickness and bite force mean values were earlier described. ${ }^{17}$

Age, gender and body variables had no effects on crossbite tendency in both dentition phases (Table 2). In the early mixed dentition, children with a higher AFH/FWB ratio were significantly more likely to have functional posterior crossbite, after controlling the mentioned variables.

The correlations of FWB and FWI with masseter thickness were significantly positive, while AFH/ FWB and AFH/FWI showed negative correlation with masseter and temporal muscle thickness in all groups (Table 3). Bite force was negatively correlated with AFH/FWB in the MCB group. AFH did not correlate with bite force or muscle thickness in any group. No significant correlations in the DNO group were observed.

\section{Discussion}

There is interaction between craniofacial dimensions and jaw muscle size in adults and growing individuals. ${ }^{8,12,18,19}$ The temporal and masseter muscle volumes exert influence on the size of their adjacent skeletal sites - such as temporal fossa, zygomatic arch and mandibular ramus - and on which muscle force is exerted..$^{18}$ Thus, the postural asymmetry that exists in the presence of malocclusion ${ }^{20}$ may contribute to differences in muscle thickness, ${ }^{1}$ masticatory function ${ }^{2,6}$ and skeletal growth and development. ${ }^{3,5}$ 
Table 1 - Minimal (min) and maximal (max) values for masseter (Mm) and temporal (Tm) muscle thickness (mm) and bite force $(\mathrm{BF})(\mathrm{N})$.

\begin{tabular}{|c|c|c|c|c|c|c|c|c|c|c|}
\hline \multirow{2}{*}{\multicolumn{3}{|c|}{$\begin{array}{l}\text { Group } \\
\text { Side }\end{array}$}} & \multicolumn{2}{|c|}{$\mathrm{DNO}(\mathrm{n}=15)$} & \multicolumn{2}{|c|}{$\mathrm{DCB}(\mathrm{n}=10)$} & \multicolumn{2}{|c|}{$\mathrm{MNO}(\mathrm{n}=13)$} & \multicolumn{2}{|c|}{$M C B(n=11)$} \\
\hline & & & Left & Right & $\mathrm{N}$ & $C B$ & Left & Right & $\mathrm{N}$ & $C B$ \\
\hline \multirow{4}{*}{$\mathrm{Mm}$} & \multirow{2}{*}{ RE } & $\min$ & 7.53 & 8.37 & 8.30 & 8.77 & 8.33 & 8.33 & 8.23 & 7.97 \\
\hline & & $\max$ & 10.57 & 10.65 & 11.37 & 10.90 & 11.93 & 11.43 & 11.90 & 11.87 \\
\hline & \multirow{2}{*}{$\mathrm{Ml}$} & $\min$ & 8.80 & 9.80 & 9.73 & 9.70 & 9.77 & 10.13 & 9.60 & 9.67 \\
\hline & & $\max$ & 12.83 & 12.80 & 13.60 & 13.23 & 14.30 & 14.37 & 13.63 & 13.17 \\
\hline \multirow{4}{*}{$\operatorname{Tm}$} & \multirow{2}{*}{ RE } & $\min$ & 2.37 & 2.20 & 2.17 & 2.27 & 2.37 & 2.43 & 2.13 & 2.27 \\
\hline & & $\max$ & 2.87 & 2.73 & 2.93 & 3.00 & 3.17 & 3.07 & 2.83 & 2.87 \\
\hline & \multirow{2}{*}{$\mathrm{Ml}$} & $\min$ & 3.07 & 3.03 & 2.80 & 3.10 & 2.70 & 2.83 & 2.47 & 2.67 \\
\hline & & $\max$ & 3.90 & 4.10 & 3.63 & 3.90 & 4.10 & 3.93 & 3.90 & 3.80 \\
\hline \multirow{2}{*}{\multicolumn{2}{|c|}{ BF }} & $\min$ & \multicolumn{2}{|c|}{126.15} & \multicolumn{2}{|c|}{130.36} & \multicolumn{2}{|c|}{206.05} & \multicolumn{2}{|c|}{114.14} \\
\hline & & $\max$ & \multicolumn{2}{|c|}{239.69} & \multicolumn{2}{|c|}{260.72} & \multicolumn{2}{|c|}{294.36} & \multicolumn{2}{|c|}{235.49} \\
\hline
\end{tabular}

RE: resting; MI: maximal intercuspation; CB: crossbite-side; N: normal side; DNO: deciduous-normal occlusion; DCB: deciduous-crossbite; MNO: mixednormal occlusion; MCB: mixed-crossbite.

Table 2 - Descriptive statistics and multivariable logistic regression significances (LoR).

\begin{tabular}{|c|c|c|c|c|c|c|c|}
\hline \multirow{2}{*}{ Group } & \multirow{2}{*}{ Age (months) } & \multicolumn{2}{|c|}{ Gender (n) } & \multirow{2}{*}{ Weight (kg) } & \multirow{2}{*}{ Height (m) } & \multirow{2}{*}{ AFH/FWB } & \multirow{2}{*}{ AFH/FWI } \\
\hline & & q & $\hat{0}$ & & & & \\
\hline DNO & $58.67 \pm 8.85$ & 5 & 10 & $20.12 \pm 4.20$ & $1.09 \pm 0.07$ & $0.76 \pm 0.03$ & $0.99 \pm 0.05$ \\
\hline $\mathrm{DCB}$ & $60.50 \pm 8.00$ & 4 & 6 & $20.39 \pm 4.04$ & $1.10 \pm 0.07$ & $0.76 \pm 0.02$ & $0.99 \pm 0.06$ \\
\hline LoR & $\mathrm{ns}$ & \multicolumn{2}{|c|}{$\mathrm{ns}$} & ns & ns & $\mathrm{ns}$ & ns \\
\hline MNO & $72.85 \pm 6.09$ & 6 & 7 & $25.90 \pm 4.44$ & $1.20 \pm 0.05$ & $0.76 \pm 0.03$ & $1.00 \pm 0.05$ \\
\hline MCB & $71.91 \pm 4.70$ & 8 & 3 & $23.72 \pm 7.00$ & $1.17 \pm 0.06$ & $0.78 \pm 0.04$ & $0.98 \pm 0.04$ \\
\hline LoR & $\mathrm{ns}$ & \multicolumn{2}{|c|}{ ns } & $\mathrm{ns}$ & ns & * & $\mathrm{ns}$ \\
\hline
\end{tabular}

AFH: anterior facial height; FWB: bizygomatic facial width; FWI: intergonial width; DNO: deciduous-normal occlusion; DCB: deciduous-crossbite; MNO: mixed-normal occlusion; MCB: mixed-crossbite; ${ }^{*} p<0.05$; ns: not significant.

Positive correlations between masseter thickness and FWB and FWI were found, whereas AFH/FWB and $\mathrm{AFH} / \mathrm{FWI}$ ratios determined negative correlations, indicating that children with strong or thick masseter had wider transverse head dimensions in proportion to their AFH. Adults and growing individuals with larger faces ${ }^{8,12}$ and wider maxillary dental $\operatorname{arch}^{1}$ presented thicker masseter than those with a long-face pattern. However, in the current study the temporal thickness did not correlate with facial dimensions. Lateral cephalometry has shown that masseter thickness correlated negatively with mandibular inclination, and positively with mandibular ramus height in adults. ${ }^{21}$ The masseter thick- ness and facial morphology measurements in growing individuals demonstrated that the thickness was negatively related with AFH and mandibular length, and positively with FWB and FWI. ${ }^{8,12}$ In the present research, AFH showed no significant correlation with thickness, agreeing with adult findings. ${ }^{19}$ Studies with young children were not found for comparisons.

There were no differences between boys and girls in group comparisons, and no gender influence for crossbite tendency (Tables 1 and 2), probably because gender differences become significant at puberty. ${ }^{2,8,13}$ Otherwise, it has been considered that bite force is influenced by age and gender, ${ }^{13,14,22}$ de- 
Table 3 - Pearson's coefficients $(r)(p<0.05)$ among facial dimensions, masseter $(\mathrm{Mm})$ and temporal (Tm) muscle thickness, and bite force $(B F)$.

\begin{tabular}{|c|c|c|c|c|c|c|}
\hline \multirow{2}{*}{ Variables } & \multicolumn{2}{|c|}{ Group DCB } & \multicolumn{2}{|c|}{ Group MNO } & \multicolumn{2}{|c|}{ Group MCB } \\
\hline & Side & r & Side & r & Side & r \\
\hline FWB $\times$ Mm RE & CB & 0.71 & Right & 0.57 & - & - \\
\hline $\mathrm{FWI} \times \mathrm{Mm} \mathrm{MI}$ & $\mathrm{CB}$ & 0.62 & - & - & $\mathrm{N}$ & 0.60 \\
\hline $\mathrm{FWI} \times \mathrm{Mm} \mathrm{RE}$ & $\mathrm{CB}$ & 0.63 & - & - & CB & 0.60 \\
\hline $\mathrm{AFH} / \mathrm{FWB} \times \mathrm{Mm} \mathrm{MI}$ & $\mathrm{N} / \mathrm{CB}$ & $-0.65 /-0.67$ & Left & -0.56 & - & - \\
\hline $\mathrm{AFH} / \mathrm{FWI} \times \mathrm{Mm} \mathrm{MI}$ & N & -0.63 & - & - & CB & -0.68 \\
\hline $\mathrm{AFH} / \mathrm{FWB} \times \mathrm{Mm}$ RE & - & - & Left & -0.66 & - & - \\
\hline $\mathrm{AFH} / \mathrm{FWI} \times \mathrm{Mm}$ RE & - & - & - & - & $N$ & -0.70 \\
\hline $\mathrm{AFH} / \mathrm{FWI} \times \mathrm{Tm} \mathrm{RE}$ & $N$ & 0.68 & - & - & - & - \\
\hline AFH/FWB $\times$ Tm RE & - & - & Left & -0.60 & - & - \\
\hline $\mathrm{AFH} / \mathrm{FWB} \times \mathrm{BF}$ & - & - & - & - & - & -0.61 \\
\hline
\end{tabular}

AFH: anterior facial height; FWB: bizygomatic width; FWI: intergonial width; RE: resting; MI: maximal intercuspation; N: normal side; CB, crossbite side.

creasing with dental and periodontal diseases, temporomandibular dysfunction, and malocclusion. ${ }^{2}$ Early studies have shown that bite force magnitude was related to vertical jaw relationship, face height, and mandibular inclination, form and length. ${ }^{10,22,23}$ In the present study, the MCB group showed a significant negative correlation between bite force and AFH/FWB ratio, i.e., children with longer faces tend to present lower bite forces. This agrees with adults $^{23}$ and older children ${ }^{2,10,11,22}$ findings, as strong masticatory muscle action was associated with a tendency to parallelism between the jaws bases, short lower face height, and small gonial angle, that is, good conditions for masticatory muscle strength development. But in deciduous dentition the facial dimensions did not correlate with muscle strength, corroborating the fact that masticatory muscle function and facial form interaction can occur later. ${ }^{4,8,24}$

This study did not find association between facial morphology and malocclusion in deciduous dentition, agreeing with the findings of Katz et al.7 (2004). However, in the early mixed dentition, children with a higher AFH/FWB ratio were significantly more likely to have crossbite (Table 2). Craniofacial asymmetries may be the consequence of the malocclusion, and not only the cause. Lower face height and the maxillary-mandibular intermolar width ratio were respectively positive and negative related with crossbite in mixed dentition. ${ }^{3}$ Accordingly, the presence of a small effective maxillary to mandibular skeletal width ratio would suggest a skeletal crossbite component and it would be a reason for early intervention. Untreated crossbite in childhood might lead to progressive asymmetric compensation of the condyle-fossa relationship and results in a positional deviation of the mandible and a dentoalveolar asymmetry, ${ }^{20,25}$ which maintain the crossbite occlusion in adults. ${ }^{25}$

A long-face pattern in early mixed dentition was significantly associated with a lower bite force and a higher probability for those children to develop crossbite. As bite force and jaw elevator muscles are some of the components of the chewing function, they can affect the face dimensions. ${ }^{22}$ The decrease in bite force magnitude may reflect on orofacial structure growth and development, ${ }^{2}$ and the abnormal movement of the mandible (mandibular shift) may contribute to adverse effects as well. ${ }^{1,5}$

Furthermore, inadequate growth of craniofacial structures and dentofacial complex results from several genetic and environmental factors, for example oral breathing. ${ }^{4}$ However, the correct diagnosis of oral breathing is controversial due to the definition criteria used, which are often subjective. The lack of straightforwardness of the exams may result in incorrect diagnostics and consequently in inadequate 
treatment. ${ }^{4}$ Thus, mild oral breathing was not taken into consideration on sample selection, since craniofacial morphology and dental patterns are affected by severe oral breathing sustained for long periods during high potential growth spurs. ${ }^{16}$ The children of the studied sample did not have their full potential growth yet, due to their age, as considered by other studies. ${ }^{16,24}$

Despite the reliable results found, the sample size could be considered a limitation of this study, but the rationale of early treatment is to get the full benefit of the high plasticity of the facial skeletons; so the recognition of malocclusions should be considered as soon as possible. ${ }^{5,6}$ Therefore, further studies in young children are required to evaluate craniofacial growth and development in the presence of

\section{References}

1. Kiliaridis S, Katsaros C, Raadsheer MC, Mahboubi PH. Bilateral masseter muscle thickness in growing individuals with unilateral crossbites [abstract]. J Dent Res. 2000;79:497.

2. Sonnesen L, Bakke M, Solow B. Bite force in pre-orthodontic children with unilateral crossbite. Eur J Orthod. 2001;23(6):741-9.

3. Allen D, Rebellato J, Sheats R, Ceron AM. Skeletal and dental contributions to posterior crossbites. Angle Orthod. 2003;73(5):515-24

4. Lessa FC, Enoki C, Feres MF, Valera FC, Lima WT, Matsumoto MA. Breathing mode influence in craniofacial development. Rev Bras Otorrinolaringol. 2005;71(2):156-60.

5. Pinto AS, Buschang PH, Throckmorton GS, Chen P. Morphological and positional asymmetries of young children with functional unilateral posterior crossbite. Am J Orthod Dentofacial Orthop. 2001;120(5):513-20.

6. Saitoh I, Hayasaki H, Iwase Y, Nakata M. Improvement in jaw motion following treatment of unilateral crossbite in a child with primary dentition: a case report. J Craniomandib Pract. 2002;20(2):129-34.

7. Katz CRT, Rosenblatt A, Gondim PPC. Nonnutritive sucking habits in Brazilian children: effects on deciduous dentition and relationship with facial morphology. Am J Orthod Dentofacial Orthop. 2004;126(1):53-7.

8. Raadsheer MC, Kiliaridis S, Van Eijden TM, Van Ginkel FC, Prahl-Andersen B. Masseter muscle thickness in growing individuals and its relation to facial morphology. Arch Oral Biol. 1996;41(4):323-32.

9. Raadsheer MC, van Eijden TM, van Ginkel FC, Prahl-Andersen B. Contribution of jaw muscle size and craniofacial malocclusion, to assess early treatment benefits.

\section{Conclusion}

In the studied sample, masticatory muscle thickness was related to face width, and children in the early mixed dentition with a long-face tendency presented lower bite force and higher probability to present functional posterior crossbite. Age, gender, and body variables were not influencing factors on predicting functional posterior crossbite in deciduous and early mixed dentition.

\section{Acknowledgments}

We are grateful to Mr. L. Ferrua for his help in statistical analysis. This study was supported by FAPESP-01/10442-3.

morphology to human bite force magnitude. J Dent Res. 1999;78(1):31-42.

10. García-Morales P, Buschang PH, Throckmorton GS, English JD. Maximum bite force, muscle efficiency and mechanical advantage in children with vertical growth patterns. Eur J Orthod. 2003;25(3):265-72.

11. Ingervall B, Minder C. Correlation between maximum bite force and facial morphology in children. Angle Orthod. 1997;67(6):415-22.

12. Kiliaridis S, Kalebo P. Masseter muscle thickness measured by ultrasonography and its relation to facial morphology. J Dent Res. 1991;70(9):1262-5.

13. Kiliaridis S, Kjellberg H, Wenneberg B, Engstrom C. The relationship between maximal bite force, bite force endurance, and facial morphology during growth. A cross-sectional study. Acta Odontol Scand. 1993;51(5):323-31.

14. Kiliaridis S, Georgiakaki I, Katsaros C. Masseter muscle thickness and maxillary dental arch width. Eur J Orthod. 2003;25(3):259-63.

15. Oueis H, Ono Y, Takagi Y. Prediction of mandibular growth in Japanese children age 4 to 9 years. Pediatr Dent. 2002;24(3):264-8.

16. Lyle KC. Airway compromise and dentofacial abnormalities. J Gen Orthod. 2000;11(4):9-18.

17. Castelo PM, Gavião MB, Pereira LJ, Bonjardim LR. Masticatory muscle thickness, bite force and occlusal contacts in young children with unilateral posterior crossbite. Eur J Orthod. 2007;29(2):149-56.

18. Kitai N, Fujii Y, Murakami S, Furukawa S, Kreiborg S, Takada K. Human masticatory muscle volume and zygomatico-man- 
dibular form in adults with mandibular prognathism. J Dent Res. 2002;81(11):752-6.

19. van Spronsen PH, Weijs WA, Valk J, Prahl-Andersen B, van Ginkel FC. Relationships between jaw muscle cross-sections and craniofacial morphology in normal adults, studied with magnetic resonance imaging. Eur J Orthod. 1991;13(5):35161.

20. Myers DR, Barenie JT, Bell RA, Williamson EH. Condylar position in children with functional posterior crossbites: before and after crossbite correction. Pediatr Dent. 1980;2(3):1904.

21. Kubota M, Nakano H, Sanjo I, Satoh K, Sanjo T, Kamegai T et al. Maxillofacial morphology and masseter muscle thickness in adults. Eur J Orthod. 1998;20(5):535-42.
22. Sonnesen L, Bakke M. Molar bite force in relation to occlusion, craniofacial dimensions, and head posture in pre-orthodontic children. Eur J Orthod. 2005;27(1):58-63.

23. Kovero O, Hurmerinta K, Zepa I, Huggare J, Nissinen M, Kononen M. Maximal bite force and its association with spinal posture and craniofacial morphology in young adults. Acta Odontol Scand. 2002;60(6):365-9.

24. Defabjanis P. Impact of nasal airway obstruction on dentofacial development and sleep disturbances in children: preliminary notes. J Clin Pediatr Dent. 2003;27(2):95-100.

25. Langberg BJ, Arai K, Miner RM. Transverse skeletal and dental asymmetry in adults with unilateral lingual posterior crossbite. Am J Orthod Dentofacial Orthop. 2005;127(1):615 . 